\title{
The Augmented Blood Pressure Response to Chronic Angiotensin II Infusion in Partially Nephrectomized Rats
}

\author{
Minoru Yasujima, Masayuki Kanazawa*, Kazunori \\ Yoshida*, Masahiro Kohzuki*, Hiroshi Watanabe, \\ Masao Hiwatari, Tokutaro Sato and Keishi Abe* \\ Institute of Rehabilitation Medicine and *the Second \\ Department of Internal Medicine, Tohoku University School \\ of Medicine, Narugo 989-68 and Sendai 980
}

Yasujima, M., Kanazawa, M., Yoshida, K., Kohzuki, M., Watanabe, H., Hiwatari, M., Sato, T. and Abe, K. The Augmented Blood Pressure Response to Chronic Angiotensin II Infusion in Partially Nephrectomized Rats. Tohoku J. Exp. Med., 1993, 169 (4), 271-277 — To assess potential roles of circulating levels of angiotensin II (Ang II) in the regulation of blood pressure in rats with reduced functional renal mass, we studied the blood pressure response to chronic infusion of Ang II at a subpressor dose in partially nephrectomized rats. Male SpragueDawley rats were subjected to $1 / 6,2 / 6,3 / 6,4 / 6$ and $5 / 6$ nephrectomy, and sham operation a week before the infusion of Ang II at a rate of $400 \mu \mathrm{g} / \mathrm{kg} /$ day delivered intraperitoneally by osmotic minipumps for 14 days, or vehicle alone served as controls. Infusion of Ang II was associated with a slight but significant increase in systolic blood pressure in $1 / 6$ nephrectomized rats $(p<0.05)$, whereas it did not induce a significant change in systolic blood pressure in sham-operated rats. Hypertension during Ang II infusion was more prominent in 2/6 nephrectomized rats than that in $1 / 6$ nephrectomized rats $(p<0.05)$. However, there was no significant difference in hypertension during Ang II infusion among groups in 2/6, $3 / 6,4 / 6$ and $5 / 6$ nephrectomized rats. Blood urea nitrogen and serum creatinine levels, endogenous creatinine clearance, urinary excretion of protein as indices of renal function, and urinary sodium excretion were not affected by chronic infusion of Ang II in 1/6,2/6,3/6,4/6 and 5/6 nephrectomized, and sham-operated rats. There was also no significant difference in circulating levels of Ang II among Ang II infused-groups in $1 / 6$ and $2 / 6$ nephrectomized rats. These results indicate that elevated levels of circulating Ang II are capable of causing a sustained hypertension in rats with reduced functional renal mass. In addition, they also suggest that circulating levels of Ang II could play a more important role in the regulation of blood pressure in rats with incipient impairments of renal function.

Ang II; renal impairment; nephrectomized rats; blood pressure regulation; renal hypertension

Received February 18, 1993; revision accepted for publication March 5, 1993.

Request for reprints: Minoru Yasujima, M.D., Institute of Rehabilitation Medicine, Tohoku University School of Medcine, Narugo 989-68, Japan. 
It is well known that blood pressure is much more salt-sensitive when functional renal mass is reduced by a disease (Langton et al. 1963). Several reports indicate that increased renal perfusion pressure is essential for maintaining sodium balance in the face of impaired renal excretory capability such as reduced functional renal mass (Hall et al. 1984, 1986). Suppression of angiotensin II (Ang II) formation may help to minimize increased blood pressure when sodium intake is high in reduced renal mass (Hall et al. 1992). However, the quantitative importance between reduced renal mass and circulating levels of Ang II in stabilizing blood pressure has not been elucidated, particularly within physiological ranges of circulating Ang II.

In the present study, therefore, to assess the quantitative importance of reduced renal mass in stabilizing blood pressure response to the elevated levels of circulating Ang II, we studied the blood pressure response to chronic infusion of Ang II at a subpressor dose in rats with subtotal nephrectomy in a gradedreduction manner.

\section{Materials and Methods}

Male Sprague-Dawley rats, 8 week-old, were subjected to $1 / 6$ nephrectomy by infarction of one-third of the right kidney, $2 / 6$ nephrectomy by infarction of two-thirds of the right kidney, $3 / 6$ nephrectomy by the removal of the right kidney, $4 / 6$ nephrectomy by infarction of one-third of the right kidney and removal of the left kidney, 5/6 nephrectomy by infarction of two-thirds of the right kidney and removal of the left kidney, and sham operation. These subtotal nephrectomy were performed under ether anesthesia. The right kidney was exposed via middle abdominal incision and the one or two poles of the right kidney were excised by encircling them with loops of ligatures and tightening the loops. In $4 / 6$ and $5 / 6$ nephrectomized rats 5 days after the infarction of the right kidney, the left kidney was removed in total. Rats were housed in a metabolic cage disigned to prevent feces-urine contact (Model ST: Sugiyamagen, Tokyo) in order to collect samplings for determination of urine volume, urinary creatinine excretion, urinary protein excretion and urinary sodium excretion. The rats were fed a regular diet (sodium $0.24 \%$; potassium $0.69 \%$; Oriental CMF, Oriental Yeast, Tokyo) and free access to tap water. Seven days after the surgery, Ang II at a rate of $400 \mu \mathrm{g} / \mathrm{kg} /$ day was administered intraperitoneally.

$\left[\mathrm{Ile}^{5}\right]$ Ang II (Peptide Institute Inc., Osaka) was dissolved in $0.01 \mathrm{~N}$ acetic acid and delivered intraperitoneally by osmotic minipumps (Alzet 2002, Palo Alto, Ca, USA) placed in the abdominal cavity through a midline incision while the rats were under ether anesthesia. In a previous study, we assessed the effect of continuous Ang II infusion 10, 75, 100 and $150 \mathrm{ng} / \mathrm{min}$ for up to 6 days in conscious rats (Yasujima et al. 1986a). Systolic blood pressure increased dose-dependently at Ang II levels of 100 and $150 \mathrm{ng} / \mathrm{min}$, whereas the changes induced by 10 and $75 \mathrm{ng} / \mathrm{min}$ did not differ from those induced by vehicle infusion. In preliminary experiments, we confirmed that continuous infusion of Ang II at a rate of $75 \mathrm{ng} / \mathrm{min}$ for 14 days did not significantly affect the blood pressure in rats. The infusion dose at a rate of $400 \mu \mathrm{g} / \mathrm{kg} /$ day $(56 \mathrm{ng} / \mathrm{min}$ ) was then chosen as a subpressor dose.

Assuming that Ang II did not degrade during the study and the pumps dispersed fluid at a preset rate of a $0.5 \mu \mathrm{l} / \mathrm{h}$, the infusion rate of Ang II was calculated. The stability of Ang II in the osmotic minipumps was examined by comparing the blood pressure-elevating activities in the solutions recovered from the minipumps in the rats after 14 days, with those of freshly dissolved Ang II in a rat bioassay system administered intravenously as a bolus. No difference was observed in the activities between the fresh preparation and the solution 
recovered from the minipumps. Systolic blood pressure in rats was recorded daily by the indirect tail-cuff method (UEDA UR 1000, Ueda Industries Co., Tokyo) without anesthesia (Ikeda et al. 1991). The systolic blood pressure measured by this method correlated well with direct systolic blood pressure (Ikeda et al. 1991). At 14 days after the initiation of Ang II infusion, they were killed by decapitation under light ether anesthesia and trunk blood was collected in polyethylene tubes for determination of blood urea nitrogen, serum creatinine levels, and circulating Ang II levels measured in 1/6, 2/6 nephrectomized and sham operated rats. The daily body weight, fluid intake and urine volume were also determined gravimetrically. Urinary protein was measured by the colorimetric method (Tonein-TP, Otsuka Pharmaceut. Co., Tokyo). Blood urea nitrogen, serum and urinary creatinine, and urinary sodium were measured by standard autoanalyzer. Endogenous creatinine clearance was calculated by the obtained serum creatinine level and urinary creatinine excretion. Plasma Ang II was radioimmunologically determined on an extract of plasma by a modification of Boyd et al. (1969), and its validity has been previously determined (Yasujima et al. 1986b).

Statistical analysis of the data between groups was performed by two way analysis of variance for repeated measurements. Statistically significant difference on each day were isolated by the unpaired $t$-test (between groups). Values are given in terms of mean \pm s.E.

\section{Results}

Infusion of Ang II at a rate of $400 \mu \mathrm{g} / \mathrm{kg} /$ day or vehicle alone as controls during the whole experimental period were all tolerated in all experimental groups of rats. Body weight, systolic blood pressure, urine volume and urinary sodium excretion were not significantly different between the groups prior to the commencing of infusion in each experimental group of rats (Table 1).

Infusion of Ang II $(400 \mu \mathrm{g} / \mathrm{kg} /$ day) was associated with a slight but significant increase in systolic blood pressure in $1 / 6,2 / 6,3 / 6,4 / 6$ and $5 / 6$ nephrectomized rats $(p<0.05$ compared to vehicle alone in each group of rats, respectively) (Figs. 1,2), whereas it did not induce any significant change in systolic blood pressure in sham operated rats (Fig. 1). Hypertension during Ang
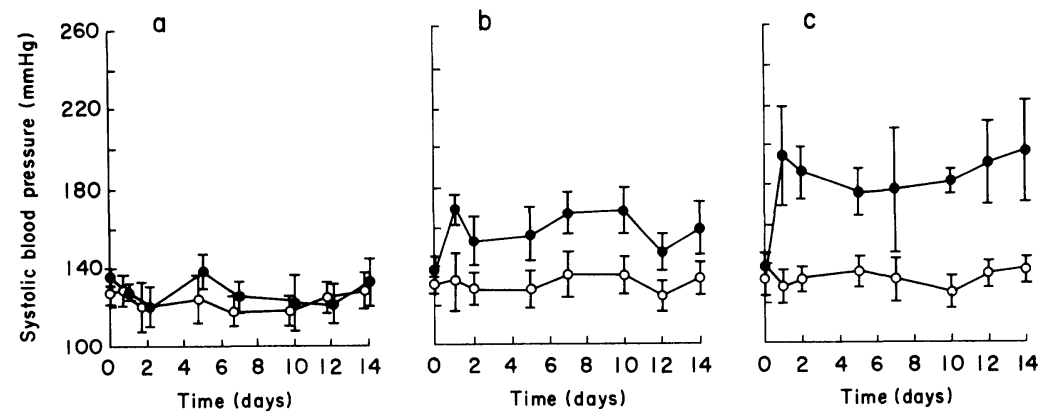

Fig. 1. Effect of chronic infusion of Ang II on systolic blood pressure in sham operated (a), and $1 / 6$ (b) and $2 / 6$ (c) nephrectomized rats. Daily systolic blood pressure in rats infused with Ang II at a rate of $400 \mu \mathrm{g} / \mathrm{kg} /$ day $(\bullet)$ and with vehicle alone as controls ( ). Results are means \pm s.e. Analysis of variance for repeated measurements revealed a significant change in systolic blood pressure in the group given Ang II $(p<0.05)$ compared to that in the group given vehicle alone in $1 / 6$ and $2 / 6$ nephrectomized rats. 

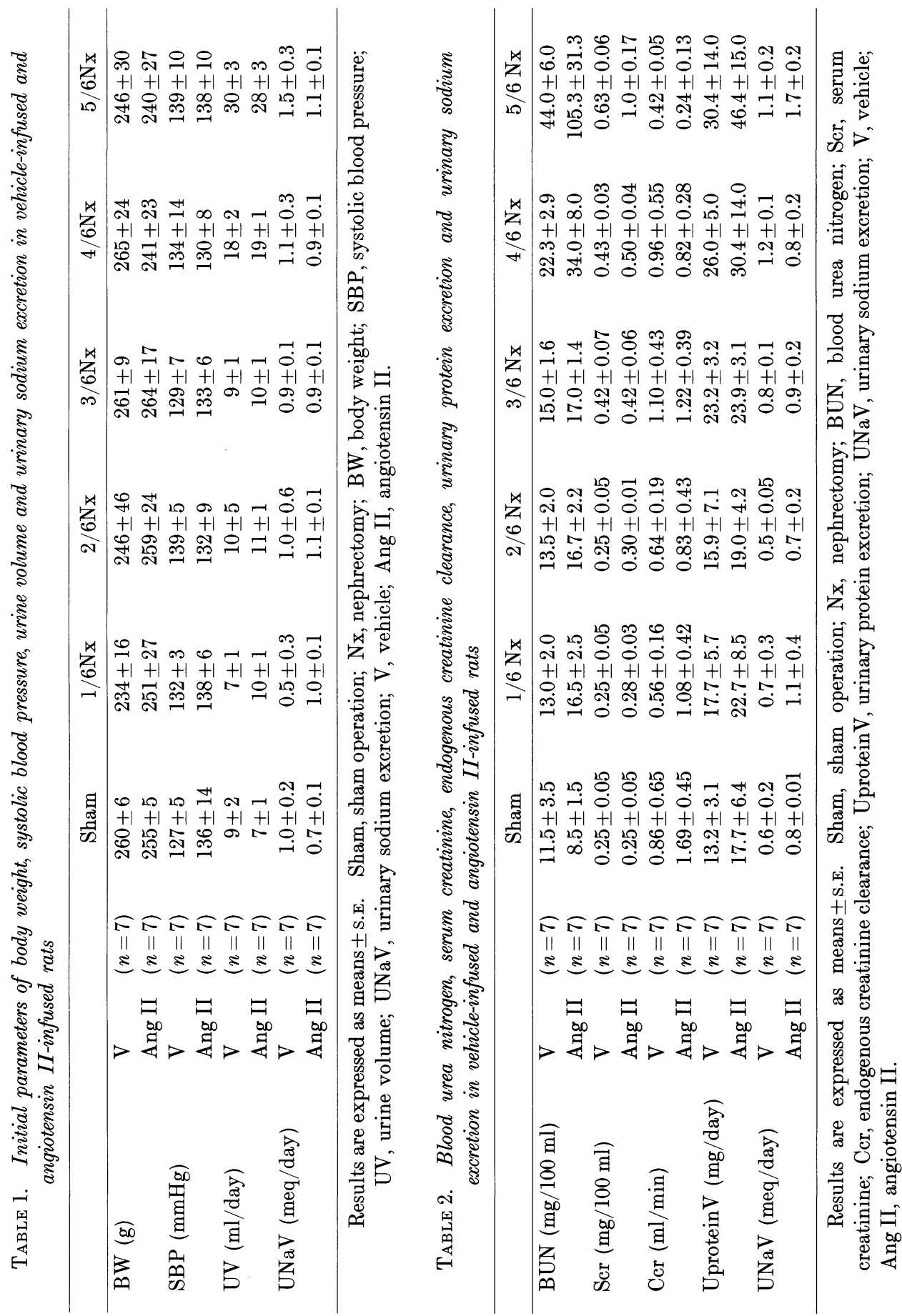

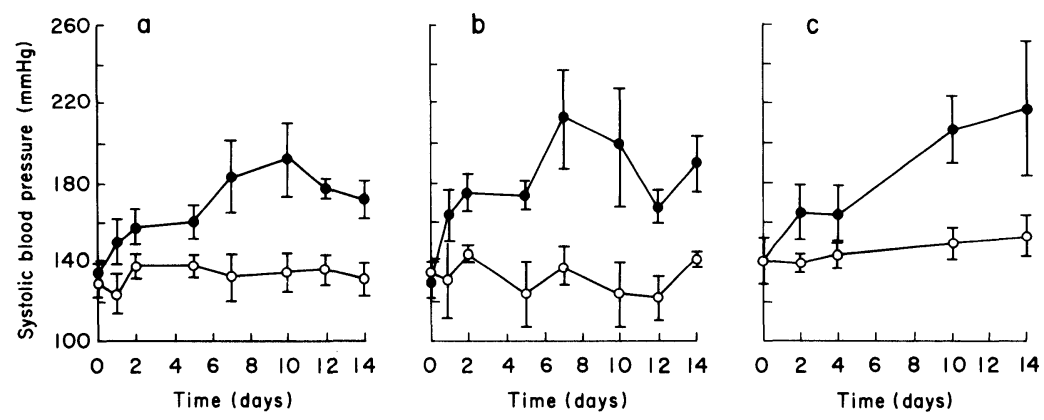

Fig. 2. Effect of chronic infusion of Ang II on systolic blood pressure in 3/6 (a), $4 / 6$ (b) and 5/6 (c) nephrectomized rats. Daily systolic blood pressure in rats infused with Ang II at a rate of $400 \mu \mathrm{g} / \mathrm{kg} /$ day $(\bullet)$ and with vehicle alone as controls (). Results are means \pm s.e. Analysis of variance for repeated measurements revealed a significant change in systolic blood pressure in the group given Ang II $(p<0.05)$ compared to that in the group given vehicle alone in $3 / 6,4 / 6$ and $5 / 6$ nephrectomized rats.

II infusion was more prominent in $2 / 6$ nephrectomized rats than that in $1 / 6$ nephrectomized rats $(p<0.05)$. However, there was no difference in hypertension during Ang II infusion among groups in 2/6, 3/6, 4/6 and 5/6 nephrectomized rats (Figs. 1, 2).

As shown in Table 2, blood urea nitrogen and serum creatinine levels, endogenous creatinine clearance, urinary excretion of protein as indices of renal function and urinary sodium excretion were not affected by chronic infusion of Ang II in each subgroup of rats. The plasma Ang II concentration was 53.0 \pm 4.4 $\mathrm{pg} / \mathrm{ml}$ in sham operated rats, and chronic Ang II infusion induced a $20.9 \mathrm{pg} / \mathrm{ml}$ increase in the absolute value of circulating level of Ang II compared to that of vehicle-infused rats. In $1 / 6$ and $2 / 6$ nephrectomized rats, chronic infusion of Ang II induced $24.4 \mathrm{pg} / \mathrm{ml}$ and $28.5 \mathrm{pg} / \mathrm{ml}$ increases, respectively, and these responses were not different among sham operated, and $1 / 6$ and $2 / 6$ nephrectomized rats.

\section{Discussion}

The main findings of this study are that the rats with reduced functional renal mass are susceptible to the hypertensinogenic effect of Ang II even when sodium is not loaded, and that its susceptibility is found even in $1 / 6$ nephrectomized rats associated with incipient renal insufficiency. In addition, it is interesting to note that the susceptibility to the hypertensinogenic effect of Ang II in 2/6 nephrectomized rats is not augmented in more than $3 / 6$ nephrectomized rats. It appears that circulating levels of Ang II could play a more important role in the regulation of blood pressure in rats with incipient impairment of renal function.

In previous study (Diz et al. 1983), Ang II-induced hypertension has been shown to be attributable to only an increase in total peripheral resistance, because 
cardiac output, stroke volume, heart rate, water intake, urine volume, and sodium and potassium excretion were not changed. When sustained Ang II infusion is combined with the graded reduction of functional renal mass, the hypertensinogenic response is augmented with no observable variations in body weight, urine volume and urinary sodium excretion.

The present observation is correlated with previous reports by Hall et al. $(1984,1986)$ that in hypertension caused by infusion of Ang II, an antinatriuretic hormone, increased renal perfusion pressure is essential for maintaining sodium balance in the face of impaired renal excretory capability. At the moment, the quantitative importance of the graded reduction of functional renal mass in maintaining sodium balance during Ang II infusion is still remained to be elucidated.

There are several potential mechanisms by which reduced functional renal mass could effectively augment the hypertensinogenic effect of Ang II infusion. Because any difference in circulating levels of Ang II between intact and 1/6 nephrectomized rats, could not be found, and the difference in the hypertensinogenic effect of Ang II also could not be found between 2/6 and more than 3/ 6 nephrectomized rats, it could be ruled out that the augmented hypertensinogenic action of Ang II in rats with reduced functional renal mass is due to only the enhanced levels of circulating Ang II associated with its impaired degradation. One of possibilities could be impaired renal excretory capability in rats with reduced functional renal mass, followed by increased renal perfusion pressure for maintaining sodium balance, although in the present experiment we could not detect impairments of renal function evaluated by levels of blood urea nitrogen and serum creatinine, and endogenous creatinine clearance in $2 / 6$ nephrectomized rats. The lack for the more prominent hypertensinogenic effect of Ang II in more than $3 / 6$ nephrectomized rats, compared to that in $2 / 6$ nephrectomized rats may be explained by recruitment of additional mechanisms to raise sodium excretion that may not have been activated under normal conditions (DeWardener and Clarkson 1985; Brenner et al. 1990; Yasujima et al. 1991). The greater impaired renal excretory capability associated with more than $2 / 6$ nephrectomy and maintaining Ang II constant would be expected to cause higher levels of various natriuretic factors, such as atrial natriuretic factors. Our previous report has shown that this natriuretic substance modulate the hypertensinogenic action induced by chronic infusion of Ang II (Yasujima et al. 1986a). Unfortunately, we cannot determine the contribution of these natriuretic hormones in maintaining sodium balance in the present studies, since we did not measure their plasma concentrations. Additinal studies are required to investigate this possibility.

In summary, chronic infusion of Ang II at a subpressor dose determined in intact rats, produced a significant elevation of blood pressure in more than $1 / 6$ nephrectomized rats, associated with no changes in body weight, urine volume and urinary sodium excretion. The magnitude of elevation of blood pressure was not 
significantly different among more than $2 / 6$ nephrectomized rats. Thus, these results indicate that even rats with incipient renal insufficiency is susceptible to the hypertensinogenic effect of Ang II.

\section{Acknowledgments}

This study was partly supported by Grants for Scientific Research No. 04670511 and No. 0477052 from the Ministry of Health and Welfare, Japan, and from the Miyagi Prefectural Kidney Association, Japan. We wish to acknowledge the excellent technical assis-. tance of Miss Naomi Sato.

\section{References}

1) Boyd, G.W., Landon, J. \& Peart, C.S. (1969) Radioimmunoassay of angiotensin II. Proc. R. Soc. Lond. (Biol.), 173, 327-338.

2) Brenner, B.M., Ballermann, B.J., Gunning, M.E. \& Zeidel, M.L. (1990) Diverse biological actions of atrial natriuretic peptide. Physiol. Rev., 70, 666-699.

3) DeWardener, H.E. \& Clarkson, M. (1985) Concept of natriuretic hormone. Physiol. Rev., 65, 658-759.

4) Diz, D.I., Baer, P.G. \& Nasjletti, A. (1983) Angiotensin II-induced hypertension in the rat: Effects on the plasma concentration, renal excretion, and tissue release of prostaglandins. $J$ Clin. Invent., 72, 466-477.

5) Hall, J.E., Granger, J.P., Hester, R.L., Coleman, T.G., Smith, M.J., Jr. \& Cross, R.B. (1984) Mechanism of escape from sodium retention during angiotensin II hypertension. Am. J. Physiol., 246, F627-F634.

6) Hall, J.E., Granger, J.P., Hester, R.L. \& Montani, J.P. (1986) Mechanisms of sodium balance in hypertension: Role of pressure natriuresis. J. Hypertens., 4, Suppl. 4, S57-S65.

7) Hall, J.P., Mizelle, H.L., Brands, M.W. \& Hildebrandt, D.A. (1992) Pressure natriuresis and angiotensin II in reduced kidney mass, salt-induced hypertension. $A m$. J. Physiol., 262, R61-R71.

8) Ikeda, K., Nara, Y. \& Yamori, Y. (1991) Indirect systolic and mean blood pressure determination by a new tail cuff method in spontaneously hypertensive rats. Lab. Anim., 25, 26-29.

9) Langton, J.B., Guyton, A.C., Douglas, B.H. \& Dorsett, P.E. (1963) Effects of changes in salt intake on arterial pressure and renal function in partially nephrectomized dogs. Circ. Res., 12, 508-513.

10) Yasujima, M., Abe, K., Kohzuki, M., Tanno, M., Kasai, Y., Sato, M., Omata, K., Kudo, K., Takeuchi, K., Hiwatari, M., Kimura, T., Yoshinaga, K. \& Inagami, T. (1986a) Effect of atrial natriuretic factor on angiotension II-induced hypertension in rats. Hypertension, 9, 748-753.

11) Yasujima, M., Abe, K., Tanno, M., Kohzuki, M., Omata, K., Kasai, Y., Kudo, K., Tsunoda, K., Sato, M., Chiba, S. \& Yoshinaga, K. (1986b) Effects of sodium and angiotensin II on urinary active and inactive kallikrein in rats. J. Hypertens., 4, 1318.

12) Yasujima, M., Abe, K., Kanazawa, M., Yoshida, K., Kohzuki, M., Sato, M., Takeuchi, K., Ota, K., Kimura, T. \& Yoshinaga, K. (1991) Atrial natriuretic polypeptide and blood pressure regulation in rats with reduced reral mass. In: Atrial and Brain Natriuretic Peptides, edited by H. Matsuo \& H. Imura, Kodansha Scientific Ltd., Tokyo, pp. 391-399. 